\title{
Somatostatin Methylation as a Biomarker for Gastric Cancer: Ready for "Prime Time" or for Further Validation?
}

\author{
Ajay Goel
}

Published online: 17 November 2010

(C) Springer Science+Business Media, LLC 2010

Gastric cancer remains the fourth leading cancer in worldwide incidence, and the second leading cause of cancer-related death. Therefore, it continues to be a global healthcare problem. Infection with Helicobacter pylori (H. pylori), a class one carcinogen according to the World Health Organization classification, is believed to be one of the major underlying risk factors for this disease. Nonetheless, other risk factors such as high-salt diet, lack of fruit intake and genetics of the host and $H$. pylori are all factors that interplay together to dictate the outcome in a population. This complexity continues to challenge our understanding of the biology of this devastating cancer [1, 2]. Over the past few decades, there has been a steady decline in the incidence of $H$. pylori infection in the United States and many Western countries. Notably, as this trend continues, there has been a significant decline in the overall incidence of noncardia gastric cancer. In the last decade, tremendous gains have been made for a better understanding of the molecular basis underlying the neoplastic transformation of the gastric epithelial cells; however, the precise molecular mechanisms operational in this disease still remain elusive. Nonetheless, in light of current epidemiological and scientific evidence, there is a general consensus that gastric carcinogenesis is a multistep process that requires equal participation of not only genetic events, but frequent involvement of epigenetic alterations as well.

Epigenetic refers to modifications of the genome heritable during cell division that do not involve changes in the DNA sequence. Epigenetic mechanisms act to change the

\footnotetext{
A. Goel $(\bowtie)$

Gastrointestinal Cancer Research Laboratory, Baylor University

Medical Center, 3500 Gaston Avenue, Suite 250 Hoblitzelle,

Dallas, TX 75246, USA

e-mail: ajayg@baylorhealth.edu
}

accessibility of the chromatin to transcriptional regulation via modifications of the DNA and by modification or re-arrangement of the nucleosomes. The role of epigenetic mechanisms, in particular DNA methylation of $\mathrm{CpG}$ dinucleotides within promoter regions of genes, in regulating the expression of genes has gained considerable attention in the past few years [3]. Promoter DNA methylation offers an alternative mechanism for silencing gene expression, irrespective of the DNA copy numbers or the mutational status of the gene [4, 5]. From a molecular standpoint, $\mathrm{CpG}$ island methylation is viewed to provide the obligatory "second hit" in the Knudson's two-hit hypothesis to inactivate the normal allele of a tumor suppressor gene.

Growing evidence suggests that aberrant DNA methylation is a critical mechanism for tumor suppressor gene inactivation in most human cancers [5]. Promoter hypermethylation has been associated with a wide variety of genes, including genes involved in cell proliferation, cell cycle, apoptosis, angiogenesis, DNA repair and metastasis. Apparently, hypermethylation-associated gene silencing provides cancer cells with a growth advantage similar to deletions and mutations. Given the unprecedented interest in identifying the molecular basis of human cancers, data on the list of hypermethylated genes in different cancers are progressively increasing.

In this issue of Digestive Diseases and Sciences, Jackson and colleagues [6] provide interesting new findings involving aberrant DNA methylation, specifically methylation of somatostatin (SST), in gastric adenocarcinoma. In this study, the authors have used state-of-the-art methodologies and demonstrated that the SST transcripts are down-regulated in $93 \%$ of gastric carcinoma samples (30 of 32 cases). Next, in their quest to understand the molecular mechanism for the abrogated SST expression in 
GC tissues, the authors demonstrated a significant increase in SST hypermethylation levels in gastric cancers compared to normal tissues, supporting their hypothesis for hypermethylation-induced loss of gene expression as one of the potential mechanisms for SST regulation in GC [6]. SST promoter hypermethylation and silencing was also detected in seven gastric cancer cells that were tested. Lastly, gastric cancer cell lines treated with 5-aza-deoxycytidine treatment showed reduced promoter DNA methylation levels of SST accompanied by restoration of its mRNA expression. The authors concluded that SST hypermethylation plays a critical role in regulating its expression in gastric cancer, and that SST hypermethylation may serve as a potential biomarker for gastric cancer.

The most provocative aspect of the findings of Jackson and colleagues [6] is that they provide potential new insights into the pathogenic mechanisms regulating gastric cancer. However, these results are not entirely surprising because similar data on SST hypermethylation has been previously reported in other gastrointestinal tumors, including colorectal cancers [7] and adenomas [8], as well as esophageal cancers [9]. Somatostatin is a known tumor suppressor, and exerts its tumor suppressive effects by multiple mechanisms, including inhibition of growth factors and hormones that have growth-promoting effects, its antiangiogenic potential, its ability to regulate the immune system which in turn helps regulate the levels of various peptides and growth factors, and lastly due to its proapoptotic potential [10]. Taken together, in the gastrointestinal tract, SST is a classic paracrine transmitter, whereby upon release it acts on adjacent cells to exert its growth inhibitory effects. In other tumors, however, SST operates through an autocrine mechanism to suppress tumor growth. In cancer therapy, SST and its analogs are widely used to treat neuroendocrine tumors where they are particularly effective in reducing hormone secretion. Interestingly, SST analogs may also reduce gastric tumor size, a concept that is currently being investigated in other gastrointestinal tumors. Because of its biological action, it is not surprising to find evidence that endogenously produced SST may inhibit tumor formation. Currently more than 1,000 clinical applications have been suggested for these pan-inhibitory SST analogs, highlighting their enormous clinical potential in the field of gastroenterology. Therefore, given the important functions of SST in the gastrointestinal tract, the findings of Jackson et al. [6], which initially may seem trivial, are actually quite critical and integral to our understanding of the role of SST in gastric carcinogenesis.

The conclusions drawn by Jackson and colleagues [6] for a cause-and-effect relationship between SST promoter hypermethylation and its inactivation in gastric cancer may ultimately be well established. Additionally, although it might be attractive to conclude that promoter hypermethylation may be a predominant mechanism of SST inactivation in gastric and other cancers, unresolved issues cloud the picture, and it is worth evaluating the data a bit more critically. The first and foremost question is whether SST methylation observed in gastric cancer tissues is a simple reflection of a hallmark "field defect" in affected gastric cancer tissues? In this regard, the authors have compared SST methylation in their tumor tissues with the histologically normal tumor-adjacent tissues (NAT) only [6]. Inclusion of methylation analyses in gastric epithelial mucosa from healthy unaffected controls would have certainly added an additional perspective to the data. In addition, since some of the NATs demonstrated down-regulation and concurrent hypermethylation of SST, analyses of healthy gastric mucosa would have facilitated a more convincing case for tumor-specificity of SST methylation in gastric cancer. Second, the authors have used a very small subset of matching cases to analyze simultaneous methylation and expression of SST. Given the small differences in SST methylation between NATs and GCs, and lack of protein expression data in these patients, the physiological significance of the present results is somewhat unclear. Therefore, data indicating that SST hypermethylation tightly linked to its protein expression in a large panel of primary cancer specimens may be helpful in the future. Lastly, for most tumor suppressor genes, DNA methylation induction involves inactivation of only one of the alleles, while the second allele is lost due to another mutational event (mutation or deletion). Data from this study did not address the issue whether SST deletions can also occur.

Epigenetic mechanism of gene inactivation, including tumor suppressor gene hypermethylation, is undoubtedly important in cancer development and represents an alternative means of inactivating tumor suppressor genes. Nevertheless, the standard of proof for establishing that hypermethylation of the promoter of any given gene has a critical role in loss of expression and cancer development should probably be set quite high, regardless of whether the gene is a well-established tumor suppressor gene, or a potential tumor suppressor gene such as SST. Although the present study did provide evidence for an association between SST hypermethylation and gene (mRNA) expression, it would be helpful if future studies can validate that SST hypermethylation is tightly linked to loss of protein expression in a large panel of primary gastric cancers.

Nevertheless, the authors should be strongly encouraged to continue in this area of research and especially to study SST methylation in patients with specific forms of gastric cancer (i.e. diffused or intestinal types, chronic gastritis cases, different grade and stage of GCs, and lastly in advanced stage tumors with metastatic potential). The next obvious question that comes to mind is whether the 
existing evidence for somatostatin methylation has the potential to serve as a biomarker for gastric cancer. The answer to the question might be "perhaps likely, but not yet ready for prime time." Even though these data clearly spark interest in this field of research, some of the enthusiasm for the conclusion for SST as a potential biomarker may be reserved until these results are independently validated in specific subsets of not only patients with gastric cancer but also patients with premalignant disease. Lastly, it would also be helpful to gather additional data on the possibility of detecting SST methylation non-invasively, in blood or fecal specimens. Such data would definitely fuel interest in a more in-depth and rigorous development of SST hypermethylation as a potential biomarker for gastric cancer. In conclusion, Jackson et al. should be commended for delving into important research, which has good potential to contribute to the understanding of gastric cancer pathogenesis and the future potential of using SST hypermethylation as a biomarker for the early detection of this disease. Until such data are gathered, some readers may simply dismiss these results as "yet another hypermethylated gene in cancer" and may not completely appreciate the true significance of this study.

Acknowledgment This study is funded by NIH Grant R01CA129286.

Conflict of interest The author has no conflicts of interest to report.

\section{References}

1. Parsonnet J, Vandersteen D, Goates J, Sibley RK, Pritikin J, Chang Y. Helicobacter pylori infection in intestinal- and diffusetype gastric adenocarcinomas. J Natl Cancer Inst. 1991;83: 640-643.

2. Watanabe T, Tada M, Nagai H, Sasaki S, Nakao M. Helicobacter pylori infection induces gastric cancer in mongolian gerbils. Gastroenterology. 1998;115:642-648.

3. Jones PA, Baylin SB. The fundamental role of epigenetic events in cancer. Nat Rev Genet. 2002;3:415-428.

4. Herman JG, Baylin SB. Gene silencing in cancer in association with promoter hypermethylation. N Engl J Med. 2003;349: 2042-2054.

5. Robertson KD. DNA methylation, methyltransferases, and cancer. Oncogene. 2001;20:3139-3155.

6. Jackson K, Soutto M, Peng D, Hu T, Marshal D, El-Rifai W. Epigenetic silencing of somatostatin in gastric cancer. Dig Dis Sci. 2010. doi:10.1007/s10620-010-1422-z.

7. Mori Y, Cai K, Cheng Y, et al. A genome-wide search identifies epigenetic silencing of somatostatin, tachykinin-1, and 5 other genes in colon cancer. Gastroenterology. 2006;131:797-808.

8. Paun BC, Kukuruga D, Jin Z, et al. Relation between normal rectal methylation, smoking status, and the presence or absence of colorectal adenomas. Cancer. 2010;116:4495-4501.

9. Jin Z, Mori Y, Hamilton JP, et al. Hypermethylation of the somatostatin promoter is a common, early event in human esophageal carcinogenesis. Cancer. 2008;112:43-49.

10. Reubi JC, Laissue JA. Multiple actions of somatostatin in neoplastic disease. Trends Pharmacol Sci. 1995;16:110-115. 